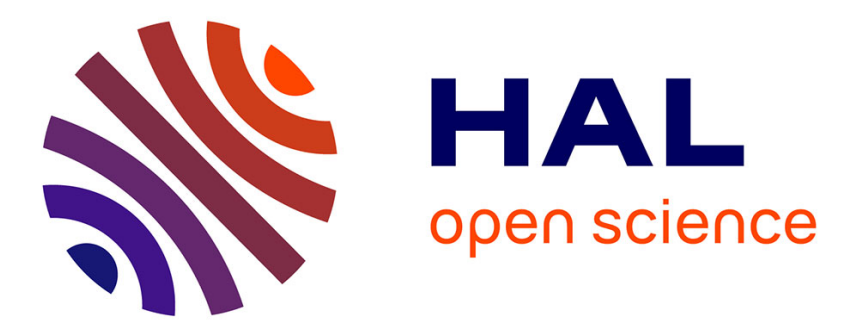

\title{
Bien-être au travail et qualité de vie des enseignants : quelles différences selon l'ancienneté ?
}

\author{
L. Zavidovique, Franck Gilbert, M.-N. N Vercambre-Jacquot
}

\section{To cite this version:}

L. Zavidovique, Franck Gilbert, M.-N. N Vercambre-Jacquot. Bien-être au travail et qualité de vie des enseignants : quelles différences selon l'ancienneté ?. Archives des Maladies Professionnelles et de L'Environnement, 2018, 79 (2), pp.105-119. 10.1016/j.admp.2017.09.005 . hal-01985025

\section{HAL Id: hal-01985025 \\ https://hal.science/hal-01985025}

Submitted on 17 Jan 2019

HAL is a multi-disciplinary open access archive for the deposit and dissemination of scientific research documents, whether they are published or not. The documents may come from teaching and research institutions in France or abroad, or from public or private research centers.
L'archive ouverte pluridisciplinaire HAL, est destinée au dépôt et à la diffusion de documents scientifiques de niveau recherche, publiés ou non, émanant des établissements d'enseignement et de recherche français ou étrangers, des laboratoires publics ou privés. 
Bien-être au travail et qualité de vie des enseignants: quelles différences selon l'ancienneté ?

Teachers' well-being at work and quality of life: any differences according to years of experience?

Titre courant : ressenti des enseignants et ancienneté

L Zavidovique, F Gilbert, MN Vercambre-Jacquot

Fondation d'entreprise MGEN pour la Santé Publique

3 Square Max-Hymans

75748 Paris Cedex 15

Contact : mvercambre@mgen.fr 


\section{Summary}

Purpose of the study: Teachers are at risk of burnout. Preliminary data has suggested that age might increase this risk. The objective of this study was to evaluate, among teachers, differences of work and general well-being according to years of experience, and to suggest recommendations.

Methods: In the population-based postal survey "Qualité de vie des enseignants" ("Teachers' Quality of Life") (MGEN Foundation/Ministry of education), 2320 primary and secondary teachers were asked about their well-being at work (satisfaction with professional experience, teaching experience evolution over the past five years, three dimensions of the Maslach Burnout Inventory) and general well-being (quality of life, perceived health and four scores of the WHOQOL-BREF questionnaire). These well-being indicators were analyzed in multivariate regression models adjusted on sociodemographic and occupational factors, according to the categories of years of experience: $\leq 5,6-29, \geq 30$.

Results: Compared to more experienced teachers, early-career teachers had less favorable work conditions and a lower environmental health score $(-3$ points $95 \% \mathrm{IC}=[(-5.1)-(-1.0)]$; $\mathrm{p}=0.005)$. Senior teachers were more likely than mid-career teachers to judge their work as becoming " more and more difficult » $(\mathrm{OR}=2.6[2.0-3.4] ; \mathrm{p}<0.001)$. They also tended to show more emotional exhaustion and burnout symptomatology. They were less satisfied with their quality of life $(O R=0.7[0.5-0.9] ; p=0.009)$ and their health $(O R=0.7[0.5-0.9] ; p=0.002)$, especially from a physical and social relationship point of view (-5.4 points [(-7.1)-(-3.8)]; $p<0.001$ and -3.0 points $[(-4.8)-(-1.3)] ; p=0.001$ respectively).

Conclusion: This study adds further evidence towards the hypothesis of a decrease in teachers' well-being at the end of their career, and supports the interest of carrying out targeted 
support and preventive actions. Attention should also be paid to early-career teachers who may face particularly difficult work conditions, regardless of their inexperience.

Keywords: teacher; age; seniority / years of experience; occupational health; job satisfaction; burnout; subjective health; quality of life 


\section{Résumé}

Objectif de l'étude. L'enseignement est une profession à risque d'épuisement professionnel. Des données préliminaires suggèrent que l'âge augmenterait ce risque. L'objectif a été d'évaluer les différences de bien-être au travail et général des enseignants selon leur ancienneté et d'en déduire des préconisations.

Méthodes. Dans le cadre de l'enquête postale nationale "Qualité de vie des enseignants » (Fondation MGEN/Éducation nationale), 2320 enseignants des $1^{\text {er }}$ et $2^{\text {nd }}$ degrés ont été interrogés sur leur bien-être au travail (bilan de l'expérience professionnelle, évolution de l'exercice du métier depuis 5 ans, trois dimensions du Maslach Burnout Inventory) et général (qualité de vie, santé perçue et quatre scores du questionnaire WHOQOL-BREF). Ces indicateurs de bien-être ont été modélisés en fonction de l'ancienneté catégorisée en trois classes ( $\leq 5$ ans, 6-29 ans, $\geq 30$ ans) dans des modèles de régression ajustés sur divers facteurs sociodémographiques et professionnels.

Résultats. Par rapport aux enseignants plus expérimentés, les enseignants en début de carrière avaient des conditions d'exercice moins favorables et un score de santé environnementale moins bon $(-3$ points $95 \% \mid C=[(-5,1)-(-1,0)] ; p=0,005)$. Les enseignants en fin de carrière étaient plus susceptibles que leurs homologues en milieu de carrière de juger l'exercice du métier « de plus en plus difficile » $(O R=2,6[2,0-3,4] ; p<0,001)$. Ils tendaient aussi à présenter plus d'épuisement émotionnel et de burnout. Ils étaient moins satisfaits de leur qualité de vie $(O R=0,7[0,5-0,9] ; p=0,009)$, de leur santé $(O R=0,7[0,5-0,9] ; p=0,002)$, notamment physique $(-5,4$ points $[(-7,1)-(-3,8)] ; p<0,001)$ et sociale $(-3,0$ points $[(-4,8)-(-1,3)]$; $p=0,001)$ 
Discussion. Cette étude plaide en faveur d'un affaiblissement du bien-être des enseignants en fin de carrière et appuie l'intérêt de réaliser des actions de prévention et d'accompagnement ciblées. Une attention doit aussi être portée aux enseignants en début de carrière qui peuvent être confrontés à des contextes particulièrement difficiles malgré leur inexpérience.

Mots clés : enseignant ; âge ; ancienneté ; santé au travail ; satisfaction professionnelle ; épuisement professionnel ; ressenti ; qualité de vie 


\section{INTRODUCTION}

Parmi les fonctionnaires et assimilés, les enseignants partagent une même mission de service public, celle de l'éducation des générations futures. Au-delà de cette relative homogénéité liée à l'objectif commun, ces personnels - nombreux : environ 850000 enseignants des $1^{\text {er }}$ et $2^{\text {nd }}$ degrés en France [1] - sont amenés à exercer leur métier dans des environnements et des conditions divers, selon l'âge et la classe des élèves bien sûr, mais aussi la taille de l'établissement ou encore son contexte social et géographique.

Les contraintes qui placent ce métier, encore très souvent vécu solitairement, parmi ceux particulièrement à risque d'épuisement professionnel [2] sont multiples [3] : représentation face à la classe sans possibilités de retrait ou d'évitement, complexification avec les programmes changeants [4], classes chargées [3], absence de césure entre les tâches professionnelles et familiales $[5,6]$, interrelations parfois tendues avec divers publics pouvant aller jusqu'à la violence et ses conséquences psychologiques [7], manque de reconnaissance sociale [8, 9], important engagement personnel et implication émotionnelle. À ces sources de stress vient se superposer un facteur susceptible de moduler notablement le vécu professionnel : l'ancienneté dans le métier.

Quelques études ont attiré l'attention sur les débuts et fins de carrière des enseignants pointant des risques spécifiques chez les jeunes enseignants [10] et une certaine érosion de la motivation professionnelle, parfois source de rupture tant sur le plan professionnel que personnel chez les plus âgés [9]. Cependant, les données épidémiologiques sur le sujet, récentes ou plus anciennes, restent rares et peu consensuelles. Ainsi, bien que le vieillissement aille généralement de pair avec un état de santé moins bon, des études notent une tendance linéaire à l'augmentation avec l'âge de la satisfaction au travail chez les salariés 
en général [11-13], et plus spécifiquement chez les enseignants [14, 15]. D’autres études ne retrouvent pas d'association entre l'ancienneté et la satisfaction au travail [16-19]. Certaines études concluent au contraire que l'effet de l'ancienneté sur le bien-être, y compris des enseignants, n'est pas linéaire, les milieux de carrière bénéficiant d'une meilleure satisfaction que les plus jeunes et les plus anciens [20,21]. Plusieurs éléments pourraient expliquer ces différences : protocoles d'études hétérogènes avec des indicateurs de satisfaction au travail ou de bien-être variés, prise en compte de l'âge ou de l'ancienneté et recours ou non à la catégorisation, grande variabilité des professions étudiées donc des contextes socioéconomiques et culturels et des itinéraires professionnels sous-jacents.

En France, les enseignants, fonctionnaires, bénéficient d'une grande stabilité d'emploi. Cependant, dans un contexte de réformes de leur formation d'une part et d'augmentation de l'âge de la retraite d'autre part, alors même que la médecine préventive dans la fonction publique dispose de moyens limités [22-24], il nous a semblé judicieux d'évaluer le bien-être au travail et le bien-être général des enseignants selon leur ancienneté dans la profession. Ainsi, notre objectif a été de rechercher, par comparaison aux enseignants en milieu de carrière, les spécificités de conditions de travail et de bien-être des enseignants débutants d'une part, des enseignants en fin de parcours professionnel d'autre part. La finalité de cette démarche est de contribuer à identifier des points de vigilance pour la médecine préventive, les ressources humaines et l'ensemble des acteurs de prévention de l'Éducation nationale afin de proposer des préconisations en vue d'un meilleur accompagnement des enseignants tout au long de leur parcours professionnel.

Dans cette optique, nous avons exploité les données de l'enquête nationale " Qualité de vie des enseignants ॥. En effet, cette enquête à caractère représentatif avait pour but de dresser un état des lieux de la santé des enseignants en France en lien avec leurs conditions de travail 
et comportait, en plus de l'information sur l'ancienneté dans le métier, des sections dévolues à l'évaluation du bien-être au travail et du bien-être général. 


\section{METHODE}

\section{Enquête "Qualité de vie des enseignants »}

L'enquête "Qualité de vie des enseignants» (QVE) a été élaborée par la Fondation d'entreprise MGEN pour la santé publique [25]. Suite à l'obtention des autorisations légales, elle a été mise en œuvre entre avril et juillet 2013 avec l'appui technique de la Direction de l'évaluation de la prospective et de la performance (DEPP) du Ministère de l'Éducation Nationale, de l'Enseignement Supérieur et de la Recherche (MENESR). Le dispositif d'enquête a consisté en un auto-questionnaire de 20 pages (caractéristiques sociodémographiques, conditions de travail, santé et qualité de vie), envoyé avec relance à un échantillon de 5000 enseignants des $1^{\text {er }}$ degré, $2^{\text {nd }}$ degré et du supérieur, et complété par des informations administratives (corps professionnel, modalité de service, caractéristiques de l'établissement d'affectation principale, épisodes de congés maladie) issues des bases de données de gestion des personnels de l’Éducation nationale.

\section{Population d'étude : les enseignants des $1^{\mathrm{er}}$ et $2^{\text {nd }}$ degrés}

Les 5000 enseignants concernés par l'enquête ont été tirés au sort avec une stratification sur le sexe, la classe d'âge, le type d'établissement et le secteur d'enseignement croisé avec l'appartenance à l'éducation prioritaire (public hors prioritaire/public prioritaire/privé sous contrat) dans l'annuaire des personnels du MENESR. Parmi ces 5000 personnes, 2653 ont retourné un questionnaire exploitable (taux de réponse brut de 53\%). Cet échantillon de répondants a été redressé par pondération afin de retrouver une structure de population semblable à l'ensemble des enseignants en France pour les variables de stratification. Dans la présente étude, nous avons exclu de l'échantillon d'analyse les 255 enseignants du supérieur ayant répondu à l'enquête QVE eu égard à leurs spécificités professionnelles, notamment leur 
cursus et leurs risques psychosociaux [2]. Nous avons également exclu 78 enseignants n'ayant pas précisé leur année de début de carrière, laissant un échantillon d'analyse de 2320 enseignants des $1^{\text {er }}$ et $2^{\text {nd }}$ degrés.

\section{Définition de l'ancienneté et corrélation avec l'âge}

Pour chaque enseignant, nous avons défini l'ancienneté dans le métier comme la période de temps écoulée entre l'année d'entrée dans l'enseignement et l'année de l'enquête. L'année d'entrée dans l'enseignement a été déterminée à partir de la question « En quelle année avezvous commencé votre carrière d'enseignant(e) (titulaire ou non titulaire) ? ». À noter que I'ancienneté ainsi définie peut se distinguer du nombre d'années d'exercice du fait d'éventuels détachements, césures, disponibilités, congés parentaux, congés maladie ou de longue durée. Cependant l'écart ne concerne qu'un petit nombre, n'excédant généralement pas quelques années. Cette notion d'ancienneté est ainsi très liée à l'âge dans cette profession à l'itinéraire particulièrement stable, comme l'avait confirmé une analyse préliminaire (corrélation de Pearson $=0,90 ; p<0,001)$.

Dans la mesure où les associations entre avancée dans la carrière et bien-être professionnel peuvent être non linéaires [21], nous avons étudié l'ancienneté en la catégorisant en trois classes « a priori » : $\leq 5$ ans (" début de carrière »), 6-29 ans (" milieu de carrière »), $\geq 30$ ans (« fin de carrière »). En fait, la littérature disponible dans le champ ne permettait pas de dégager un consensus pour définir formellement le début et la fin de carrière, la catégorisation des tranches d'âge ou d'ancienneté étant hétérogènes d'un article à l'autre. Notre choix d'une limite de début de carrière à 5 ans permettait de considérer des enseignants encore en rodage et présentant ainsi des risques spécifiques au début de carrière, tout en étant en nombre suffisant pour l'analyse statistique. La catégorie " fin de carrière " démarrant à 30 ans 
d'ancienneté permettait de considérer des enseignants à risque d'émoussement de la motivation professionnelle du fait d'un long parcours dans cette profession. De plus, ces derniers avaient pour la quasi-totalité un âge supérieur à 50 ans, limite souvent choisie de tranche supérieure dans les études en santé au travail considérant l'âge comme variable.

Pour tester la robustesse de nos résultats en regard de notre catégorisation de l'ancienneté, des analyses de sensibilité ont été conduites, notamment avec l'âge en trois classes ( $<35$ ans ; 35-50 ans ; >50 ans), et, afin de gagner en puissance sur la catégorie des débuts de carrière, en étendant la première tranche « $\leq 5$ ans » à « $\leq 8$ ans » d'ancienneté.

Dans tous les modèles, la catégorie d'ancienneté intermédiaire "milieu de carrière » a été choisie comme catégorie de référence.

\section{Indicateurs de bien-être}

\section{Bien-être au travail}

Nous avons pris en considération cinq indicateurs de bien-être ou mal-être au travail évalués dans l'enquête QVE. Une première question s'intéressait à la satisfaction globale vis-à-vis de son expérience professionnelle d'enseignant depuis le début de carrière : « Aujourd'hui, si vous deviez faire le bilan de votre expérience professionnelle d'enseignant(e), vous diriezvous... ? ». Nous avons regroupé les réponses en deux catégories positive/négative : " assez » ou "très satisfait " versus " assez " ou " très insatisfait ». Une autre question interrogeait l'enseignant sur son ressenti vis-à-vis de l'évolution récente de l'exercice du métier : «De même, diriez-vous que depuis 5 ans (ou depuis votre première affectation si vous êtes en poste depuis moins de 5 ans), l'exercice du métier a été pour vous... ? ". Nous avons également regroupé les réponses en deux catégories positive/négative : « ni plus ni moins » ou « de moins en moins difficile » versus « de plus en plus difficile ». Enfin, le Maslach Burnout 
Inventory $(\mathrm{MBI})[26,27]$ évaluait trois dimensions du syndrome d'épuisement professionnel. Dans le champ des professions engagées dans une relation d'aide telles que les professions médicales, sociales et éducatives, le terme de "burnout " est utilisé pour désigner un syndrome d'épuisement des ressources physiques et mentales consécutif aux exigences relationnelles du contexte professionnel. Souvent utilisé pour mesurer le burnout des professions médicales, le MBI a fait l'objet d'une adaptation pour la profession enseignante [28]. Le questionnaire a également été validé en langue française [29]. II comporte 22 items de type échelle de Likert s'intéressant tour à tour à l'épuisement émotionnel (score/54), c'està-dire une impression de fatigue et de saturation affective et émotionnelle; la dépersonnalisation (score/30) qui se traduit pour l'enseignant par une représentation négative des élèves et une déshumanisation de la relation affective; le faible accomplissement personnel au travail (score/48) qui correspond à un sentiment d'incompétence, de perte d'autorité et d'échec vis-à-vis, par exemple, de la réussite des élèves. Dans les modèles et pour respecter l'approche préconisée par Maslach [26], chacun des trois scores a été dichotomisé en isolant le tertile "péjoratif », à savoir : épuisement émotionnel fort, dépersonnalisation forte et faible accomplissement professionnel. Dans une analyse complémentaire, nous avons modélisé la probabilité de " burnout ", événement que nous avons défini comme le fait de présenter systématiquement des scores péjoratifs pour les trois échelles du MBI.

\section{Bien-être général}

Nous avons considéré six indicateurs de bien-être général, tous issus de la version française de l'auto-questionnaire abrégé World Health Organisation - Quality of Life (WHOQOL-BREF) [30] qui permet d'évaluer la santé au sens défini par l'OMS : " un état de complet bien-être 
physique, mental et social ". Cette version abrégée en 26 items s'ouvre sur deux items évaluant respectivement la qualité de vie ressentie et la santé subjective. Nous avons regroupé les réponses en deux catégories positive/négative, identifiant ainsi respectivement les enseignants estimant avoir une « bonne » ou " très bonne » qualité de vie versus une « très mauvaise ", " mauvaise " ou " ni bonne ni mauvaise " qualité de vie et ceux " satisfait » ou « très satisfait » de leur santé versus ceux " pas du tout satisfait ", " pas satisfait » ou " ni satisfait ni insatisfait » de leur santé. Viennent ensuite 24 items permettant d'approfondir quatre facteurs de la qualité de vie (score ramené systématiquement sur 100) : la santé physique (énergie, douleur, fatigue, dépendance, capacité de travail) ; la santé psychologique (sentiments négatifs et positifs, estime de soi, mémoire et concentration); les relations sociales (relations personnelles, support social) ; l'environnement (ressources financières, sécurité, habitat, transport).

\section{Facteurs de confusion potentiels dans l'étude des liens entre ancienneté et bien-être}

En tant que facteurs de confusion potentiels, nous avons considéré les différentes variables disponibles dans l'enquête QVE pouvant être associées à la fois à l'ancienneté et au bien-être. Nous avons ainsi pris en compte 1 ) des facteurs personnels : sexe (homme/femme), situation familiale selon le statut marital et la présence d'enfant(s) au foyer (couple avec enfant(s)/couple sans enfant(s)/seul(e) avec enfant(s)/seul(e) sans enfant(s)) ; 2) des facteurs individuels caractérisant le travail: type d'établissement d'enseignement (maternelle/élémentaire/collège/lycée d’enseignement général et technologique/lycée professionnel), temps de travail moyen déclaré hors enseignement (en heures), temps partiel (oui/non), statut professionnel (statutaire/vacataire ou contractuel), décharge de travail (oui/non), nombre d'établissement(s) d'enseignement (un/deux ou plus), temps de trajet 
domicile-travail ( $\leq 15 \mathrm{~min} / 16-30 \mathrm{~min} />30 \mathrm{~min}$ ) ; 3) des facteurs caractérisant l'établissement d'enseignement : taille de l'établissement (<200 élèves/200-1000 élèves/>1000 élèves), zone d'implantation de l'établissement selon l'Insee (commune rurale/ville isolée/centre d'agglomération/banlieue), indicateur d'origine sociale majoritaire des élèves (favorisée/moyenne/défavorisée) ; 4) des facteurs caractérisant les risques psychosociaux au travail : l'exposition depuis le début de l'année scolaire à la violence psychologique au travail selon la définition du questionnaire de Leymann [31], à savoir des « comportements hostiles de la part d'une ou plusieurs personne(s) de l'environnement de travail qui visent de manière continue et répétée à blesser, oppresser, maltraiter, ou encore exclure ou isoler pendant une longue période de temps » (ni témoin ni victime de violence/témoin uniquement/victime), les appréciations des relations avec les élèves, les collègues, la direction d'établissement et les personnels administratifs respectivement réparties en deux catégories (plutôt positive/plutôt négative) et enfin les trois scores évaluant le risque psychosocial au sens du modèle de Karasek [32] : latitude décisionnelle (score/96), demande psychologique (score/36) et soutien social au travail (score/32).

\section{Traitement des valeurs manquantes pour les facteurs de confusions potentiels}

Afin d'optimiser la taille de l'échantillon final et considérant que le taux de valeurs manquantes pour les facteurs de confusion potentiels n'excédait jamais $5 \%$ de l'échantillon, nous avons procédé à une imputation avant utilisation dans les modèles multivariés. Pour les facteurs catégoriels, nous avons imputé aux valeurs manquantes la valeur la plus représentée. Pour les facteurs numériques (risques psychosociaux de Karasek notamment), nous avons imputé la moyenne du score sur la population d'étude. Des analyses de sensibilité ont été conduites dans les sous-échantillons n'ayant aucune donnée manquante. 


\section{Analyse statistique}

Après avoir décrit les caractéristiques principales de notre échantillon redressé, nous avons testé les associations bivariées entre l'ancienneté et les différents facteurs de confusion potentiels. Nous avons ensuite réalisé, pour chacun des indicateurs de bien-être au travail et de bien-être général, deux modèles de régression (régression linéaire pour les quatre scores du WHOQOL-BREF ou logistique pour les autres variables de bien-être au travail ou général qui sont dichotomiques) : un modèle univarié et un modèle ajusté sur l'ensemble des facteurs de confusion potentiels. Ont été considérés comme facteurs de confusion potentiels ceux liés avec l'ancienneté au seuil de signification $p<0,20$ (test du Chi ${ }^{2}$ pour les variables catégorielles et test de l'analyse de la variance (Fisher) pour les variables continues). Les décharges d'enseignement et le statut, très fortement liées à l'ancienneté, n'ont pas été inclus dans cette liste pour éviter tout risque de sur-ajustement. A contrario, la latitude décisionnelle, bien que non liée à l'ancienneté au seuil de 0,20, a été « forcée » dans la liste des facteurs d'ajustement aux côtés des deux autres scores de Karasek, qui eux remplissaient le critère. Nous avons par ailleurs réalisé des analyses de sensibilité notamment avec un modèle de régression linéaire sur les scores du MBI en continu. Nous avons enfin effectué des analyses d'interaction entre l'ancienneté et le fait d'avoir exercé ou non un autre métier d'une part, et le sexe d'autre part. Le cas échéant, des analyses stratifiées ont permis d'explorer les possibles effets d'interaction détectés. Les analyses ont été faites à l'aide du logiciel STATA ${ }^{\circledR}$ SE 13.0. 


\section{RESULTATS}

Après redressement, notre échantillon de 2320 individus était bien à l'image de la population enseignante française des $1^{\text {er }}$ et $2^{\text {nd }}$ degrés en 2013 en ce qui concerne ses caractéristiques sociodémographiques et professionnelles principales [33]. Par exemple, l'échantillon comprenait deux tiers de femmes et un tiers d'hommes ce qui est retrouvé à l'échelle de l'Éducation nationale. Par ailleurs, plus de la moitié des enseignants vivaient en couple avec des enfants au foyer (57\%) et environ un tiers (34\%) avait déjà exercé un autre métier (données non présentées dans les tableaux).

\section{Association entre l’ancienneté et les facteurs sociodémographiques et professionnels}

Comme attendu pour un métier stable bénéficiant du statut de fonctionnaire, l'âge et I'ancienneté dans l'enseignement étaient étroitement liés (figure 1) : hormis quelques cas de reconversion professionnelle "sur le tard ", la très grande majorité des enseignants ayant moins de 5 ans d'ancienneté étaient aussi âgés de moins de 35 ans. Par ailleurs, l'intégralité des enseignants ayant plus de 30 ans d'ancienneté avaient 48 ans ou plus. En considérant les variables statistiquement différentes en fonction de l'ancienneté au seuil $p<0,05$, nous avons pu caractériser les enseignants en début et en fin de carrière (tableau I). Les enseignants en début de carrière ( $\leq 5$ ans d'ancienneté) étaient en proportion plus nombreux à avoir exercé un autre métier et surreprésentés en élémentaire ou au collège, leur temps de trajet était en moyenne plus long, ils exerçaient plus souvent un emploi contractuel ou vacataire et étaient plus nombreux à travailler dans plusieurs établissements, en banlieue et avec des élèves d’origine sociale défavorisée. Les enseignants en fin de carrière ( $\geq 30$ ans d'ancienneté), quant à eux, enseignaient plus souvent en maternelle et lycée d'enseignement général et technologique, en centre d'agglomération, avec des élèves d'origine sociale favorisée, ils 
avaient un temps de trajet plus court en moyenne et étaient plus susceptibles d'avoir une décharge sur leur temps de travail ; enfin, ils percevaient la qualité de leurs relations aux élèves un peu moins positivement que leurs collègues ayant moins d'ancienneté.

\section{Association entre l’ancienneté et les indicateurs de bien-être au travail}

À la question « aujourd'hui, si vous deviez faire le bilan de votre expérience professionnelle d'enseignant, vous diriez vous... ? ", 25\% des enseignants en début de carrière ont répondu " très satisfait » et $60 \%$ " assez satisfait ». Ils étaient respectivement $17 \%$ et $64 \%$ parmi les enseignants en milieu de carrière et $21 \%$ et $61 \%$ parmi ceux en fin de carrière (figure $2 \mathrm{~A}$ ). Dans les modèles univarié et multivarié de cet indicateur de satisfaction professionnelle préalablement dichotomisé, les écarts avec l'ancienneté n'atteignaient pas le niveau de signification (tableau II).

Les différences en fonction de l'ancienneté étaient nettement plus marquées en ce qui concernait l'évolution de la difficulté de l'exercice du métier : $20 \%$ des enseignants en début de carrière trouvaient l'exercice du métier « de plus en plus difficile » contre $60 \%$ en milieu de carrière et $77 \%$ en fin de carrière (figure $2 \mathrm{~B}$ ). Cette tendance au pessimisme vis-à-vis de la difficulté du métier avec l'ancienneté était cohérente avec les odd-ratios très fortement significatifs estimés dans les modèles univariés et multivariés (tableau II). À caractéristiques sociodémographiques et professionnelles comparables, pour cette question, l’odd-ratio était de $0,1[0,1-0,2]$ en début de carrière, et de $2,6[2,0-3,4]$ en fin de carrière par rapport au milieu de carrière.

En ce qui concerne la symptomatologie du burnout, les médianes observées en fonction de l'ancienneté ne différaient pas sensiblement en fonction des trois groupes d'ancienneté. Dans les modèles, seule une association significative était retrouvée en univarié avec l'indicateur 
de faible accomplissement au travail, les enseignants en fin de carrière s'avérant un peu moins satisfaits sur ce point $(O R=1,3[1,0-1,6] ; p=0,038)$ (tableau II). Dans le modèle univarié, une tendance était aussi retrouvée entre ancienneté et plus forte probabilité de burnout défini comme le fait de présenter systématiquement des scores péjoratifs pour les trois échelles du MBI $(O R=1,4[1,0-2,1]$ à la limite de la significativité $-p=0,065$ - pour le contraste fin de carrière/milieu de carrière).

\section{Association entre l'ancienneté et les indicateurs de bien-être général}

Interrogés sur leur qualité de vie, $9 \%$ des enseignants en début de carrière la trouvaient « très bonne » et $6 \%$ « mauvaise ». Ils étaient respectivement $9 \%$ et $6 \%$ parmi les enseignants en milieu de carrière, et $7 \%$ et $9 \%$ parmi ceux en fin de carrière (figure $3 \mathrm{~A}$ ). La tendance à une ressenti moins positif vis à vis de la qualité de vie chez les enseignants en fin de carrière était cohérente avec l'association correspondante retrouvée dans le modèle multivarié $(O R=0,7$ $[0,5-0,9] ; p=0,009)$ (tableau III).

Par ailleurs, les enseignants en début de carrière étaient en proportion plus nombreux à se sentir en très bonne santé (19\%) comparés à leurs homologues en milieu de carrière (11\%) ou en fin de carrière (5\%) (figure 3B). Dans les modèles multivariés, la perception de l'état de santé était significativement moins satisfaisante chez les enseignants en fin de carrière $(\mathrm{OR}=0,7[0,5-0,9] ; p=0,002)$ (tableau III).

Concernant les quatre dimensions de la qualité de vie (WHOQOL-BREF), les enseignants en fin de carrière avaient en médiane un plus faible score de santé physique et de relations sociales que leurs collègues en début ou milieu de carrière, tandis que les enseignants en début de carrière avaient en médiane un plus faible score de santé environnementale que leurs collègues en milieu ou fin de carrière (figure $3 \mathrm{C}$ ). Ces résultats descriptifs étaient cohérents 
avec les modèles de régression linéaire. En effet, dans les modèles multivariés, la santé physique $(-5,4$ points $[(-7,1)-(-3,8)] ; p<0,001)$ et les relations sociales $(-3$ points $[(-4,8)-(-$ $1,3)] ; p=0,001$ ) étaient significativement moins bonnes chez les enseignants en fin de carrière par rapport à ceux en milieu de carrière. La santé environnementale était perçue comme étant moins favorable $(-3$ points $[(-5,1)-(-1,0)] ; p=0,005)$ pour les enseignants en début de carrière par rapport à ceux en milieu de carrière (tableau III). Enfin, le score de santé psychologique tendait à être un peu moins élevé (coefficient à la limite de la significativité) en fin de carrière par rapport au milieu de carrière $(-1,4$ points $[(-2,9)-0,1] ; p=0,071)$ (tableau III).

\section{Analyses de sensibilité et d'interaction}

Dans les modèles considérant comme facteur d'exposition principal l'âge en trois catégories (<35 ans; 35-50 ans; >50 ans) plutôt que l'ancienneté, les résultats étaient similaires, certaines associations étant même un peu plus marquées, notamment pour l'indicateur de bien-être social, les enseignants âgés de moins de 35 ans ayant un score statistiquement plus élevé que ceux d'âges intermédiaires (+2 points $[0,1-3,9] ; p=0,04)$. Les analyses effectuées dans l'échantillon d'enseignants ne présentant aucune valeur manquante pour les facteurs d'ajustement donnaient les mêmes résultats que ceux présentés dans les tableaux. L'analyse de l'évolution de la difficulté du métier sur les 5 dernières années après avoir exclu les enseignants en tout début de carrière conduisait aux mêmes conclusions. Si l'on étendait la première tranche d'ancienneté de moins de 5 ans à moins de 8 ans afin de gagner en puissance sur cette catégorie, les résultats retrouvés dans les modèles multivariés étaient très proches. En considérant les trois scores du MBI en continu dans des modèles de régression linéaire, les différences en fonction de l'ancienneté restaient non significatives en général. Seul le score d'épuisement émotionnel tendait à être un peu plus élevé en moyenne chez les enseignants 
en fin de carrière comparativement à ceux en milieu de carrière $(+1,1$ points $[(-0,0)-2,3]$; $p=0,056)$.

Dans l'analyse des indicateurs de bien-être au travail, aucune interaction significative n'était retrouvée entre l'ancienneté et le fait d'avoir exercé un autre métier. II n'y avait pas non plus d'argument fort en faveur d'une interaction entre le sexe et l'ancienneté vis-à-vis du bien-être professionnel, sauf en ce qui concerne la dépersonnalisation ( $p$-interaction=0,012). Pour cette dimension du burnout, une association inverse entre ancienneté et probabilité de dépersonnalisation était observée chez les hommes mais absente chez les femmes. 


\section{DISCUSSION}

Dans une enquête française à caractère représentatif réalisée en 2013 , les enseignants en début de carrière exerçaient en général dans des conditions a priori moins favorables que les enseignants plus expérimentés, mais présentaient des indicateurs de bien-être professionnel comparables. Les enseignants en fin de carrière, plus âgés, se percevaient logiquement en moins bonne santé, avaient globalement de moins bons indicateurs de qualité de vie générale, et malgré de meilleures conditions de travail générales, présentaient une tendance vers plus d'épuisement professionnel que leurs collègues en milieu de carrière.

Ces résultats doivent être commentés en tenant compte des forces et limites de la présente étude. Au nombre de ces forces se trouve son originalité, puisque c'est une des premières à évaluer le bien-être des enseignants en fonction de leur ancienneté alors même que plusieurs hypothèses contradictoires ont pu être mises en avant par ailleurs. De plus, cette analyse se fait sur un échantillon de plusieurs milliers d'enseignants, qui grâce au tirage stratifié couplé au redressement par pondération, peut être extrapolé à l'ensemble de la population enseignante française des $1^{\text {er }}$ et $2^{\text {nd }}$ degrés. Ensuite, la richesse des données disponibles dans l'enquête QVE a permis à la fois de considérer plusieurs indicateurs de bien-être complémentaires, au travail et en général, et de prendre en compte de nombreux facteurs de confusion potentiels dans les modèles statistiques. Enfin, les données professionnelles du questionnaire ont été enrichies par des informations administratives provenant des bases de données ministérielles, ce qui permet d'en renforcer la fiabilité et de limiter les données manquantes. Par contre, le dispositif d'enquête transversale par auto-questionnaire en population occupée présente inévitablement des faiblesses. Tout d'abord, la transversalité de l'étude ne permet aucune inférence causale. Ensuite, les résultats, même soigneusement 
redressés par pondération, peuvent être biaisés, notamment du fait de l'effet « travailleur sain ». En effet, les enseignants en activité et en meilleure santé sont plus susceptibles de répondre à ce type d'enquête envoyée directement sur leur lieu de travail. Cependant, sur une population d'environ 850000 enseignants des $1^{\text {er }}$ et $2^{\text {nd }}$ degré en $2013-2014$, seuls $1,9 \%$ des enseignants ont eu recours à un congé long dont une majorité d'enseignants de plus de 50 ans [34] et 715 ont démissionné [22]. Ainsi, les tendances et les différences péjoratives retrouvées chez les enseignants en fin de carrière par rapport à ceux en milieu de carrière pourraient être en réalité plus marquées. Enfin, le recueil d'informations par autoquestionnaire peut donner lieu à des erreurs de mesure ou des biais de désirabilité sociale, mais reste adapté à l'évaluation de la santé et du bien-être perçus, notamment dans une population d'enseignants à niveau d'éducation élevé, comme en témoigne la bonne acceptabilité de l'enquête (taux de retour de $53 \%$; $87 \%$ des répondants ayant émis un avis général positif sur l'enquête QVE).

Un des résultats de notre étude est que les conditions d'exercice chez les enseignants en début de carrière sont moins favorables que celles des plus expérimentés, ce qui est en accord avec le système des points qui les détermine. Bénéficiant de peu de points au terme de leur formation, les enseignants débutants se retrouvent plus souvent dans des établissements réputés difficiles [5], parfois en tant que remplaçant sur plusieurs affectations, plus fréquemment loin de leur domicile, sur plusieurs classes et/ou plusieurs établissements, ou dans des établissements d'éducation prioritaire avec généralement des emplois du temps complexes. Cependant, nos données montrent également qu'avec l'acquisition rapide d'expérience les premières années, les enseignants en début de carrière rapportent une évolution favorable concernant la difficulté du métier comparativement à leurs collègues, suggérant une bonne adaptabilité de ces jeunes professionnels. Une autre hypothèse pour 
expliquer ce résultat serait que ces jeunes enseignants manquent par définition de recul en début de carrière pour pouvoir réellement juger de la difficulté du métier et de son évolution, comme en atteste d'ailleurs la forte proportion de réponse neutre à cette question (figure 2B). Au final, le bien-être des enseignants en début de carrière et en milieu de carrière ne diffère pas statistiquement. Pour autant, et comme l'ont souligné certains auteurs pour quelquesuns, les situations d'exercice difficiles de leurs premières années peuvent être à l'origine d'une confrontation douloureuse entre les attentes et la réalité du métier d'enseignant [10, 19], source de rupture pouvant aboutir au burnout et/ou au départ de la profession enseignante. Il est possible que ces jeunes enseignants en souffrance aient moins répondu à l'enquête car de fait sortis du champ de recrutement, dépassés ou gênés vis-à-vis de leurs difficultés. Tout récemment, à partir d'autres sources de données, et même si les chiffres restent faibles au regard des effectifs totaux des personnels enseignants, on observe une augmentation des démissions des enseignants stagiaires : le nombre est ainsi passé de $65(1,1 \%)$ en 2012 à 434 $(3,2 \%)$ en 2015 [22]. Ceci pourrait témoigner de difficultés dans la formation des futurs enseignants. Prévoir un suivi rapproché de ces enseignants stagiaires et jeunes enseignants, à la fois par leur encadrement et l'inspection, pour discuter d'éventuels problèmes en classe, et par la médecine préventive en cas de besoin, semble pertinent.

A contrario, les enseignants en fin de carrière apparaissent plus installés dans leur vie, tant professionnelle que personnelle par rapport aux enseignants moins expérimentés. Pour autant, en ce qui concerne leur bien-être, un faisceau d'arguments laisse augurer d'une augmentation des difficultés chez ces enseignants chevronnés alors même qu'ils bénéficient de meilleures conditions globales de travail. Le fait que malgré un environnement a priori plus favorable, les enseignants en fin de carrière vivent moins positivement leur métier au fil des années tend à confirmer que ce dernier est pénible et donc usant indépendamment de sa 
spécificité plus intellectuelle. L'ancienneté étant très corrélée avec l'avancée en âge, les enseignants en fin de carrière présentent de moins bons indices de santé perçue, notamment physique de manière attendue, mais aussi sur le plan des relations sociales. Cela pourrait être en partie dû à l'accumulation de frustrations, d'où aussi une tendance vers une moins bonne santé psychologique. Avec l'âge, les enseignants ont également de moins en moins de ressources personnelles et de mécanismes pour faire face aux impératifs du métier. Autrement dit, le « coût » physique et psychologique du travail augmente : accumulation plus rapide de la fatigue, troubles de la voix, sensibilité accrue au bruit, moindre patience vis-à-vis des difficultés rencontrées notamment avec les élèves [9]. De plus, ce métier est solitaire institutionnellement, avec peu de collectif de travail et d'accompagnement mobilisé et peu de possibilités de changement de métier et d'évolutions hiérarchiques ou salariales. Nous pouvons émettre l'hypothèse d'une gêne pour ces enseignants à communiquer sur leurs difficultés [35] du fait d'un sentiment de honte ou de peur d'être jugés fragiles voire incompétents par leurs collègues. Par ailleurs, les enseignants en fin de carrière ont en général vécu de nombreuses évolutions de la profession et des programmes au gré des réformes, bousculant leurs habitudes et pouvant être ressenties comme en décalage avec leurs représentations du métier. Ainsi, le métier d'enseignant (conditions de travail mais aussi formation ou reconnaissance sociale) en 2013 n'est plus le même que 30 ans auparavant. Son évolution pourrait être vécue de manière péjorative avec par exemple l'émergence de problèmes relationnels avec les familles ou de discipline avec les élèves [36]. Peut en découler une érosion de la motivation et des capacités à faire face aux exigences de l'enseignement [9], et l'on comprend alors que le métier soit ressenti de plus en plus difficile à exercer. Cela peut aboutir à un sentiment d'échec vis-à-vis de la mission d'enseignement, conduisant à l'autodévalorisation du travail puis de la personne elle-même, menant enfin au burnout. Une telle 
séquence, dont la fréquence pourrait bien augmenter avec le recul de l'âge de départ à la retraite et l'allongement mécaniquement des carrières, contribuerait à expliquer la tendance observée et probablement sous-estimée dans cette étude à plus d'épuisement professionnel chez les enseignants en fin de carrière. On note d'ailleurs dans le modèle univarié un plus faible accomplissement personnel chez ces derniers. On peut ainsi émettre l'hypothèse qu'en se plaçant dans des situations d'enseignement jugées plus difficiles mais dans lesquelles les enseignants pourraient mettre à profit leurs compétences accumulées, ces derniers augmenteraient leur sentiment d'accomplissement personnel.

L'interprétation et les hypothèses précédentes conduisent à suggérer quelques préconisations, notamment pour les enseignants en fin de carrière qui, d'après notre étude, tendraient à présenter plus de mal-être au travail que leurs collègues en milieu de carrière, en plus d'une moins bonne santé. II apparaît essentiel de mettre en œuvre des mesures de prévention collectives et individuelles et d'accompagner ces enseignants en fin de carrière pour leur permettre de ne pas aggraver d'éventuels problèmes de santé du fait de leur travail, en adaptant ce travail aux enseignants. Une action efficace contre le mal-être au travail nécessite une bonne communication auprès des agents sur les moyens disponibles et l'implication conjointe de tous les acteurs du milieu de travail et de prévention en vue d'une prise en charge pluridisciplinaire coordonnée: collègues, direction d'établissement, inspection, médecin de prévention, psychologues, assistantes sociales, etc.

La priorité pour développer la prévention et le dépistage dans l'Éducation nationale avec pour objectif minimum de répondre de manière satisfaisante aux obligations règlementaires en matière de suivi des enseignants [37] est, nous semble-t-il, le recrutement de médecins de prévention. Seuls habilités à recueillir en consultation le mal-être des agents dans le respect 
absolu du secret médical et à se rendre librement dans les établissements pour observer l'organisation globale et les conditions de travail, ils représentent un pivot central dans l'analyse et l'action contre la souffrance au travail. Ainsi, le suivi des agents par un nombre suffisant de médecins de prévention permettrait à ces derniers d'anticiper et prévenir des situations de rupture professionnelle, mais également de faire remonter des cas d'organisation délétère du travail auprès de la direction, des rectorats ou encore des Comité d’Hygiène, de Sécurité et des Conditions de Travail (CHSCT). Prenant en compte les résultats de la présente étude, un exemple d'action sera d'être particulièrement attentifs à l'état de santé mentale des enseignants de plus de 30 ans d'ancienneté (ou, critère quasi équivalent, de plus de 50 ans) en leur proposant une consultation médicale comprenant une évaluation de leur bien-être au travail. Cette évaluation pourra se faire à l'aide d'un questionnaire adapté permettant aussi, en plus d'une analyse individuelle, un suivi longitudinal du bien-être au travail de la population enseignante en fin de carrière en vue d'évaluer l'efficacité des actions menées. Alertés sur des situations de travail problématiques, les médecins de prévention peuvent également agir sur le plan individuel en orientant l'agent vers son médecin traitant en vue de la prescription d'un arrêt de travail, vers un psychologue, un psychiatre, ou encore un réseau de consultation de souffrance au travail, selon les besoins et la nécessité ou non de démarrer un traitement médicamenteux et de l'extraire d'un milieu à risque pour sa santé. Cela sera favorisé par un lien étroit entre les médecins et les directions d'établissement ou administrations, notamment via la transmission des arrêts de travail et accidents imputables au service afin d'aider à identifier d'éventuels agents en difficultés. Les enseignants doivent par ailleurs être informés, par exemple par des affichages en salle des professeurs, sur la possibilité d'une consultation de médecine préventive à leur demande. Les médecins de prévention pourront en complément travailler avec les réseaux académiques de prévention, 
aide et suivi (réseaux PAS) déjà en place. Ces réseaux sont destinés à aider à la réadaptation sociale et professionnelle des agents fragilisés ou atteints de troubles psychiques, victimes d'accidents ou de maladies graves. Ces réseaux proposent, parmi d'autres actions, une cellule d'écoute confidentielle, anonyme et gratuite permettant à tout agent d'exprimer ses difficultés auprès d'un psychologue indépendant de l'Éducation nationale. Ils méritent d'être développés et une communication efficace sur leur rôle auprès des agents doit être poursuivie. De manière générale, il semblerait par ailleurs intéressant de développer un collectif de travail au travers de groupes de réflexion ou espaces de discussion entre enseignants, pouvant également impliquer la direction d'établissement ou l'inspection académique, qui seraient des lieux d'échange, de partage et de coopération, non pas seulement sur les problèmes rencontrés mais également autour de la pédagogie et des méthodes d'enseignement. Ce travail collectif, par le partage de connaissances, de compétences et de solutions face aux difficultés du métier, pourrait s'avérer une aide précieuse dans la gestion des classes d'élèves [9] et permettre de lutter de manière constructive contre la solitude liée au métier d'enseignant $[38,39]$. II pourrait aussi être proposé de réorganiser les prérogatives des inspecteurs en orientant leurs missions vers l'aide et l'accompagnement et moins vers l'évaluation individuelle et la notation qui conditionnent les avancements d'échelon et peuvent également être source de souffrances chez les enseignants. Une autre piste pour lutter contre la fragilisation des enseignants en fin de carrière serait de les faire bénéficier de plus de formations pour valider leurs acquis et diversifier leurs compétences. En favorisant parallèlement la visibilité et les possibilités de changement, provisoire ou définitif de carrière, un plus grand nombre d'enseignants en difficulté pourraient mettre ces compétences en application et bénéficier d’un détachement vers d'autres fonctions publiques, de mobilité vers l'inspection académique ou vers des 
fonctions de formation, soutien ou accompagnement de leurs collègues ou d'élèves en difficulté. Ainsi, si l'on considère conjointement le sentiment des nouveaux d'être mal préparés à des conditions d'exercice difficiles et celui des anciens d'un contact plus pénible avec les élèves, un évitement simultané des deux écueils serait que certains enseignants en difficultés en fin de carrière passent une partie de leur service à former et accompagner ceux en début de carrière. Une autre façon indirecte d'aider les enseignants fragilisés serait d'aider les élèves en difficulté, par exemple au travers du développement des Réseaux d'aides spécialisées aux élèves en difficultés (Rased) et de l’augmentation du nombre de psychologues scolaires. De plus, une rencontre systématique avec les enseignants souhaitant partir précocement à la retraite permettrait d'en connaître les raisons et de proposer des aménagements pour continuer d'exercer dans de meilleures conditions un métier qui souvent leur tient à cœur [9]. De tels aménagements (temps partiel, absences d'heures supplémentaires imposées, diversification des activités...) permettraient aux enseignants de moduler leur emploi du temps pour mieux mobiliser leurs ressources.

Au total, un meilleur suivi, un accompagnement vigilant et une surveillance accrue en fin de carrière favoriseraient une prévention efficace et le cas échéant permettraient de détecter le plus précocement possible le mal-être des enseignants. L'ensemble de ces résultats demande à être approfondi, idéalement dans un cadre longitudinal afin d'isoler les effets générationnels de ceux liés purement à l'avancée en âge. II serait également intéressant d'étudier les différences selon le sexe sur une plus grande population avec plus de puissance chez les hommes. Cela permettrait d'affiner encore les propositions d'accompagnement des enseignants aux ressources humaines et à la médecine de prévention de l’Éducation nationale. II serait par ailleurs judicieux de mettre en place des études interventionnelles visant à confirmer l'intérêt des préconisations énoncées dans cet article, ou d'évaluer des actions 
existantes: centre de réadaptation des académies d'lle-de-France, plans académiques de formation, Rased, réseaux PAS...

Conflits d'intérêts : Les auteurs déclarent ne pas avoir de conflit d'intérêt en relation avec cet article 


\section{REFERENCES}

1. Repères et références statistiques sur les enseignements, la formation et la recherche. RERS. Ministère de l'éducation nationale, Ministère de l'enseignement supérieur et de la recherche - DEPP ; 2016.

2. Jégo $S$, Guillo C: Les enseignants face aux risques psychosociaux. Comparaison des enseignants avec certains cadres du privé et de la fonction publique en 2013. Education et formation 2016, n92 (décembre 2016).

3. Ponnelle S: Contribution des déterminants personnels, organisationnels et des styles d'ajustement au stress dans l'explication de la santé subjective des enseignants du secondaire. L'orientation scolaire et professionnelle 2008(37/2):183-213.

4. Maroy C: Les évolutions du travail enseignant en France et en Europe: facteurs de changement, incidences et résistances dans l'enseignement secondaire. Revue française de pédagogie 2006(2):11-11.

5. Guedj H, Beaumont B: Personnels de l'éducation nationale: des métiers exposés aux menaces et aux insultes. Direction de l'évaluation, de la prospective, et de la performance (DEPP), vol. $n^{\circ} 25$ - juillet $2014 ; 2014$.

6. Lantheaume F: Tensions, ajustements, crise dans le travail enseignant: un métier en redéfinition. Pensée plurielle 2008(2):49-56.

7. Gilbert F, Vercambre-Jacquot M-N: Violence à l'école, violence au travail. Le cas des enseignants. Education \& formations (Ministère de l'Education Nationale - DEPP) 2016, 92:115-135.

8. Papart JP: La santé des enseignants et des éducateurs de l'enseignement primaire. Rapport à l'organisation du travail. Département de l'action sociale et de la santé ; 2003.

9. Cau-Bareille D: Vécu du travail et santé des enseignants en fin de carrière: une approche ergonomique. Rapport de recherche. Centre d'études de l'emploi ; 2009: 65. 
10. Rascle N, Bergugnat L: Les déterminants et les conséquences de l'épuisement professionnel des enseignants débutants: quels effets sur leur santé? Quels effets sur les élèves? Une recherche longitudinale de mars 2008 à septembre 2012. Université Bordeaux Segalen. http://www.cndp.fr/climatscolaire/uploads/tx_cndpclimatsco/determinants_et_consequenc es_de_lepuisement_professionnel_des_enseignants_debutants.pdf ; 2012.

11. Ng TW, Feldman DC: The relationships of age with job attitudes: a meta-analysis. Personnel Psychology 2010, 63(3):677-718.

12. Bos JT, Donders NC, Bouwman-Brouwer KM, Van der Gulden JW: Work characteristics and determinants of job satisfaction in four age groups: university employees' point of view. International Archives of Occupational and Environmental Health 2009, 82(10):1249-1259.

13. Mauno S, Ruokolainen $\mathrm{M}$, Kinnunen U: Does aging make employees more resilient to job stress? Age as a moderator in the job stressor-well-being relationship in three Finnish occupational samples. Aging \& Mental Health 2013, 17(4):411-422.

14. Lowther MA, Gill SJ, Coppard LC: Age and the determinants of teacher job satisfaction. Gerontologist 1985, 25(5):520-525.

15. Liu XS, Ramsey J: Teachers' job satisfaction: Analyses of the teacher follow-up survey in the United States for 2000-2001. Teaching and Teacher Education 2008, 24(5):1173-1184.

16. Arvidsson I, Hakansson C, Karlson B, Bjork J, Persson R: Burnout among Swedish school teachers - a cross-sectional analysis. BMC Public Health 2016, 16(1):823.

17. Pas ET, Bradshaw CP, Hershfeldt PA: Teacher-and school-level predictors of teacher efficacy and burnout: Identifying potential areas for support. Journal of school Psychology 2012, 50(1):129-145.

18. Canrinus ET, Helms-Lorenz M, Beijaard D, Buitink J, Hofman A: Self-efficacy, job satisfaction, motivation and commitment: exploring the relationships between indicators of teachers' professional identity. European journal of psychology of education 2012, 27(1):115-132. 
19. Skaalvik EM, Skaalvik S: Teacher job satisfaction and motivation to leave the teaching profession: Relations with school context, feeling of belonging, and emotional exhaustion. Teaching and teacher education 2011, 27(6):1029-1038.

20. Demirtas Z: Teachers' job satisfaction levels. Procedia-Social and Behavioral Sciences 2010, 9:1069-1073.

21. Klassen RM, Chiu MM: Effects on teachers' self-efficacy and job satisfaction: Teacher gender, years of experience, and job stress. Journal of educational Psychology 2010, 102(3):741.

22. Carle JC, Férat F: Enseignement scolaire. Sénat - Commission de la culture, de l'éducation et de la communication - $\mathrm{n}^{\circ} 144 ; 2016$.

23. Gonthier-Maurin B: Rapport d'information. Sénat - Commission de la culture, de l'éducation et de la communication - Mission d'information sur le métier d'enseignant - n601; 2012.

24. Réponse du Ministère de l'éducation nationale de l'enseignement supérieur et de la Recherche. Surveillance médicale des enseignants et personnels de l'éducation nationale tout au long de leur carrière - Question écrite n 18322. 10 Sénat du 18/02/2016. Sénat ; 2016.

25. Billaudeau N, Gilbert F, Lapie-Legouis P, Vercambre-Jacquot M-N: Enquête « Qualité de vie des enseignants " : état des lieux. 16ème Colloque de l'ADEREST 2015 ; Archives des Maladies Professionnelles et de l'Environnement ; 2015: 391-392.

26. Maslach C, Jackson S, Leiter MP: Maslach Burnout Inventory Manual. Consulting Psychological Press, Inc ; 1996.

27. Maslach C, Jackson SE: The measurement of experienced burnout. Journal of Organizational Behavior 1981, 2(2):99-113.

28. Chan DW, Hui EK: Burnout and coping among Chinese secondary school teachers in Hong Kong. British journal of educational psychology 1995, 65(1):15-25. 
29. Dion G, Tessier R: Validation de la traduction de l'Inventaire d'épuisement professionnel de Maslach et Jackson. Canadian Journal of Behavioural Science/Revue canadienne des sciences du comportement 1994, 26(2):210.

30. Baumann C, Erpelding ML, Regat S, Collin JF, Briancon S: Le questionnaire de qualité de vie WHOQOL-BREF : valeurs de références françaises des dimensions santé physique, santé psychologique et relation sociale. Revue d'epidemiologie et Sante Publique 2010, 58(1):33-

39.

31. Niedhammer I, David S, Degioanni S: La version française du questionnaire de Leymann sur la violence psychologique au travail : le "Leymann Inventory of Psychological Terror" (LIPT). Revue d'epidemiologie et Sante Publique 2006, 54(3):245-262.

32. Karasek RA: Job Demands, Job Decision Latitude, and Mental Strain: Implications for Job Redesign. Administrative Science Quarterly 1979, 24(2):285-308.

33. Repères et références statistiques sur les enseignements, la formation et la recherche. RERS. Ministère de l'éducation nationale, Ministère de l'enseignement supérieur et de la recherche - DEPP ; 2013.

34. Bilan social du Ministère de l'éducation nationale, du Ministère de l'enseignement supérieur et de la recherche - Année 2013-2014. Ministère de l'éducation nationale, Ministère de l'enseignement supérieur et de la recherche - DEPP ; 2015.

35. Vercambre MN, Brosselin P, Gilbert F, Nerriere E, Kovess-Masfety V: Individual and contextual covariates of burnout: a cross-sectional nationwide study of French teachers. BMC Public Health 2009, 9:333.

36. Meuret $D$ : La mauvaise discipline dans les classes françaises et quelques autres résultats de PISA 2015. Les notes du conseil scientifique. FCPE ; 2017.

37. Décret n $^{\circ} \mathbf{8 2 - 4 5 3}$ du 28 mai 1982 relatif à l’hygiène et à la sécurité du travail ainsi qu'à la prévention médicale dans la fonction publique - Version consolidée au 28 mars 2017 ; 2017. 
38. Dejours C: La psychodynamique du travail face à l'évaluation: de la critique à la proposition. Travailler 2011(1):15-27.

39. Dejours C: Effets de la désorganisation des collectifs sur le lien... à la tâche et à l'organisation. Revue de psychothérapie psychanalytique de groupe 2013(2):11-18. 
Tableau I : Caractéristiques des enseignants en fonction de l'ancienneté $(n=2320)$, enquête "Qualité de vie des enseignants », Fondation MGEN/Éducation nationale, 2013.

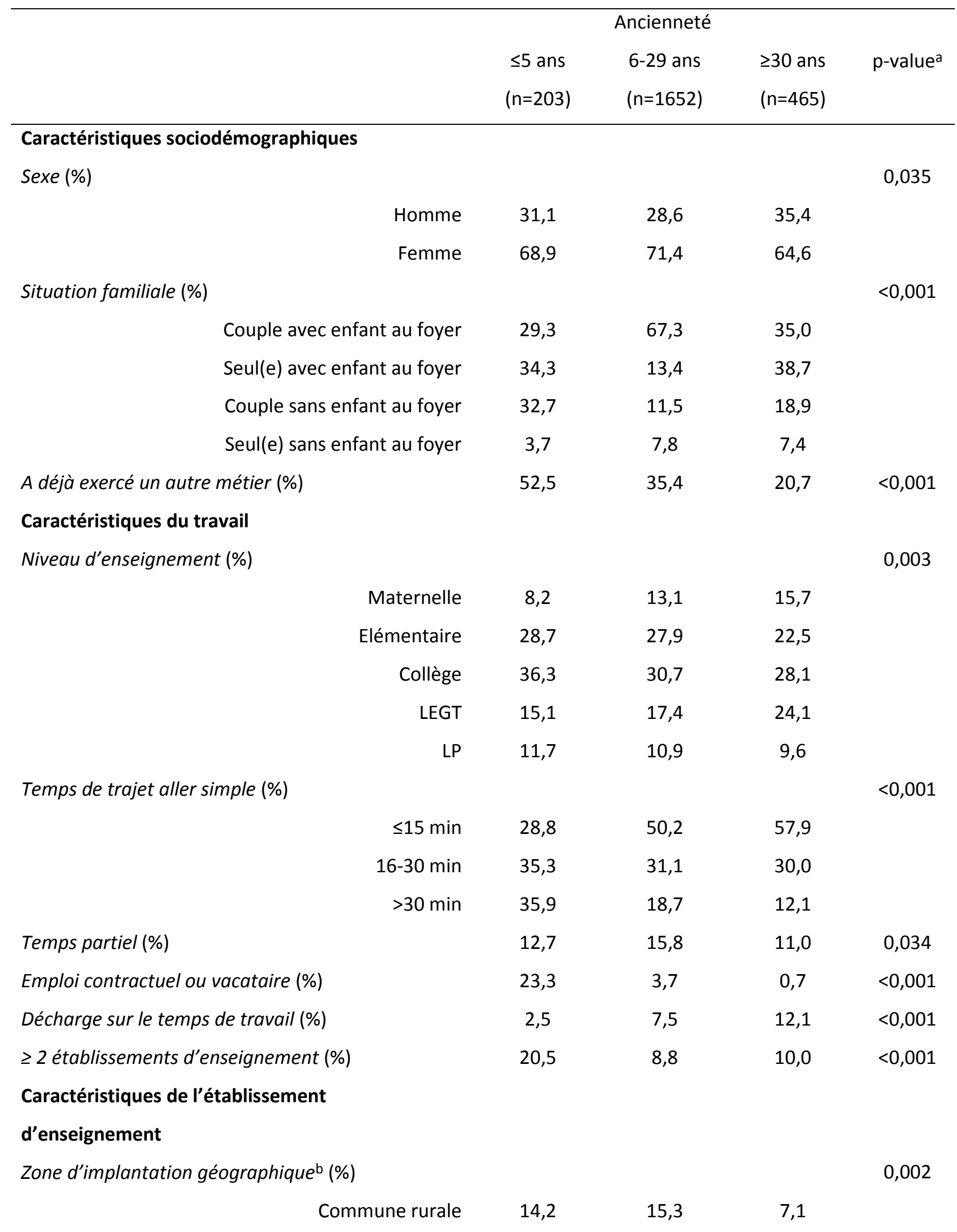




$\begin{array}{rccc}\text { Ville isolée } & 9,8 & 10,7 & 12,5 \\ \text { Centre d'agglomération } & 43,1 & 44,6 & 50,0 \\ \text { Banlieue } & 32,9 & 29,4 & 30,4\end{array}$

Taille de l'établissement

$\begin{array}{rlll}<200 \text { élèves } & 24,9 & 30,1 & 28,3 \\ 200-1000 \text { élèves } & 63,7 & 55,7 & 52,2 \\ >1000 \text { élèves } & 11,4 & 14,2 & 19,5\end{array}$

Indicateur d'origine sociale des élèves ${ }^{\mathrm{c}}(\%)$

$\begin{array}{rrrr}\text { Plutôt favorisée } & 19,3 & 23,1 & 30,3 \\ \text { Plutôt moyenne } & 46,1 & 50,8 & 49,3 \\ \text { Plutôt défavorisée } & 34,6 & 26,1 & 20,4\end{array}$

\section{Risques psychosociaux}

Scores du questionnaire de Karasek [moyenne (ET)]

$\begin{array}{rllll}\text { Latitude décisionnelle } & 76,5(7,9) & 76,0(8,0) & 75,6(8,4) & 0,333 \\ \text { Demande psychologique } & 23,5(3,7) & 23,9(4,0) & 23,3(4,1) & 0,020 \\ \text { Soutien social } & 23,1(3,6) & 22,6(3,8) & 22,3(3,9) & 0,041 \\ & & & & 0,694\end{array}$

$\begin{array}{rlll}\text { Ni victime, ni témoin } & 51,3 & 55,1 & 53,5 \\ \text { Témoin uniquement } & 31,3 & 26,9 & 27,1 \\ \text { Victime } & 17,4 & 18,0 & 19,4\end{array}$

Taux de satisfaction vis à vis des relations (\%)

$\begin{array}{rllll}\text { Avec les élèves } & 95,1 & 96,0 & 92,6 & 0,019 \\ \text { Avec les collègues } & 90,5 & 93,7 & 94,8 & 0,108 \\ \text { Avec la direction d'établissement } & 85,0 & 83,7 & 80,5 & 0,235 \\ \text { Avec les personnels administratifs } & 87,0 & 86,9 & 89,2 & 0,448\end{array}$

LEGT : lycée d'enseignement général et technologique ; LP : lycée professionnel

a Valeur de $\mathrm{p}$ du test du $\mathrm{Chi}^{2}$ pour les variables catégorielles et du test de Fisher (analyse de la variance) pour les variables continues

b Catégories Insee permettant de caractériser l'environnement urbain de l'établissement d'enseignement (http://www.insee.fr/fr/methodes/default.asp?page=definitions/unite-urbaine.htm)

c Indicateur isolant, pour le $2^{\text {nd }}$ degré, les quartiles extrêmes du ratio « nombre d'élèves d'origine plutôt favorisée / nombre d'élèves d'origine plutôt défavorisée " (données ministérielles disponibles au niveau établissement), et pour le 1 er degré, au découpage $1 / 4 \frac{1}{2} 1 / 4$ de la réponse à la question «Les élèves de l'établissement appartiennent à un milieu social plutôt...» «très défavorisé» ou «défavorisé» / «moyen» / "favorisé» ou «très favorisé»

$\mathrm{d}$ Violence psychologique selon la définition du questionnaire de Leymann : « comportements hostiles au travail visant de manière continue et répétée à blesser, oppresser, maltraiter, ou encore exclure/isoler pendant une longue période de temps » 
Tableau II : Association entre l'ancienneté et les indicateurs de bien-être au travail, enquête "Qualité de vie des enseignants ", Fondation MGEN/Éducation nationale, 2013.

\begin{tabular}{|c|c|c|c|c|c|c|}
\hline & \multicolumn{3}{|c|}{ Modèle univariéa : OR [IC95\%] } & \multicolumn{3}{|c|}{ Modèle multivariéb : OR [IC95\%] } \\
\hline & \multicolumn{3}{|c|}{ Ancienneté } & \multicolumn{3}{|c|}{ Ancienneté } \\
\hline & $\leq 5$ ans & $6-29$ ans & $\geq 30$ ans & $\leq 5$ ans & $6-29$ ans & $\geq 30$ ans \\
\hline \multicolumn{7}{|l|}{ Questions sur le bien-être professionnel } \\
\hline Bilan de l'expérience professionnelle $(n=2313)$ : & $0,7[0,5-1,1]$ & 1 (ref) & $1,0[0,7-1,2]$ & $0,8[0,5-1,3]$ & 1 (ref) & $1,0[0,7-1,4]$ \\
\hline \multicolumn{7}{|l|}{ insatisfaction } \\
\hline $\begin{array}{l}\text { Evolution du métier depuis } 5 \text { ans }(\mathrm{n}=2309) \text { : de plus en } \\
\text { plus difficile }\end{array}$ & $0,2 * * *[0,1-0,2]$ & 1 (ref) & $2,2 * * *[1,7-2,8]$ & $0,1 * * *[0,1-0,2]$ & 1 (ref) & $2,6 * * *[2,0-3,4]$ \\
\hline \multicolumn{7}{|l|}{ Symptomatologie du burnout (dimensions du MBI) } \\
\hline Epuisement émotionnel ( $n=2305)$ : fort ( $3^{\text {ème }}$ tertile) & $0,9[0,6-1,2]$ & 1 (ref) & $1,1[0,9-1,4]$ & $1,0[0,6-1,4]$ & 1 (ref) & $1,2[0,9-1,6]$ \\
\hline Dépersonnalisation ( $n=2266)$ : forte ( $3^{\text {ème }}$ tertile) & $1,2[0,9-1,6]$ & 1 (ref) & $1,0[0,8-1,3]$ & $1,2[0,8-1,7]$ & 1 (ref) & $1,0[0,8-1,3]$ \\
\hline Accomplissement personnel $(n=2259)$ : faible $\left(1^{\mathrm{er}}\right.$ tertile $)$ & $1,1[0,8-1,5]$ & 1 (ref) & $1,3 * *[1,0-1,6]$ & $1,2[0,8-1,7]$ & 1 (ref) & $1,2[0,9-1,5]$ \\
\hline Syndrome de burnout ${ }^{\complement}(\mathrm{n}=2240)$ & $0,8[04,-1,5]$ & 1 (ref) & $1,4^{*}[1,0-2,0]$ & $0,8[0,4-1,5]$ & 1 (ref) & $1,3[0,8-2,1]$ \\
\hline
\end{tabular}

${ }^{*} p \leq 0,10 ;{ }^{* *} p \leq 0,05 ; * * * p \leq 0,01$

$\mathrm{MBI}$ : Maslach Burnout Inventory

a Modèle de régression logistique univarié

b Modèle de régression logistique multivarié ajusté sur le sexe, la situation familiale, le fait d'avoir déjà exercé un autre métier, le niveau d'enseignement, le temps de trajet aller simple, le fait d'être à temps partiel, d'enseigner dans $\geq 2$ établissements d'enseignement, la zone d'implantation et la taille de l'établissement, l'indicateur d'origine sociale des élèves, les trois scores du questionnaire de Karasek (latitude décisionnelle, demande psychologique, soutien social) ainsi que le taux de satisfaction vis à vis des relations avec les élèves et avec les collègues.

${ }^{\mathrm{c}}$ Syndrome de burnout défini comme le fait de présenter systématiquement des scores péjoratifs pour les trois échelles du MBI : épuisement émotionnel $3^{\text {ème }}$ tertile, dépersonnalisation $3^{\text {ème }}$ tertile et accomplissement personnel $1^{\mathrm{er}}$ tertile. 
Tableau III : Association entre l'ancienneté et les indicateurs de bien-être général issus du questionnaire WHOQOL-BREF, enquête « Qualité de vie des enseignants », Fondation MGEN/Éducation nationale, 2013.

\begin{tabular}{|c|c|c|c|c|c|c|}
\hline & \multicolumn{3}{|c|}{ Modèle univariéa } & \multicolumn{3}{|c|}{ Modèle multivariéb } \\
\hline & \multicolumn{3}{|c|}{ Ancienneté } & \multicolumn{3}{|c|}{ Ancienneté } \\
\hline & $\leq 5$ ans & 6-29 ans & $\geq 30$ ans & $\leq 5$ ans & 6-29 ans & $\geq 30$ ans \\
\hline Questions sur le bien-être général & \multicolumn{3}{|c|}{ OR [IC95\%] } & \multicolumn{3}{|c|}{ OR [IC95\%] } \\
\hline Qualité de vie $(\mathrm{n}=2307)$ : bonne & $0,9[0,7-1,2]$ & 1 (ref) & $0,8^{*}[0,7-1,0]$ & $0,9[0,6-1,3]$ & 1 (ref) & $0,7 * * *[0,5-0,9]$ \\
\hline Santé perçue $(n=2311)$ : satisfaisante & $1,1[0,8-1,6]$ & 1 (ref) & $0,7^{* * *}[0,6-0,9]$ & $1,2[0,8-1,7]$ & 1 (ref) & $0,7 * * *[0,5-0,9]$ \\
\hline Dimensions de la qualité de vie & \multicolumn{3}{|c|}{ Coefficient [IC95\%] } & \multicolumn{3}{|c|}{ Coefficient [IC95\%] } \\
\hline Score santé physique $(\mathrm{n}=2314)$ & $0,6[(-1,5)-2,7]$ & 0 (ref) & $-4,3 * * *[(-6,1)-(-2,6)]$ & $0,3[(-1,7)-2,3]$ & 0 (ref) & $-5,4^{* * *}[(-7,1)-(-3,8)]$ \\
\hline Score santé psychologique $(\mathrm{n}=2309)$ & $0,5[(-1,5)-2,4]$ & 0 (ref) & $-1,2[(-2,7)-0,3]$ & $0,1[(-1,9)-2,0]$ & 0 (ref) & $-1,4 *[(-2,9)-0,1]$ \\
\hline Score relations sociales $(n=2308)$ & $0,2[(-2,6)-3,0]$ & 0 (ref) & $-3,4 * * *[(-5,2)-(-1,7)]$ & $0,0[(-2,8)-2,8]$ & 0 (ref) & $-3,0 * * *[(-4,8)-(-1,3)]$ \\
\hline Score environnement $(n=2315)$ & $-3,5 * * *[(-5,6)-(-1,4)]$ & 0 (ref) & $1,3 *[(-0,1)-2,7]$ & $-3,0 * * *[(-5,1)-(-1,0)]$ & 0 (ref) & $0,1[(-1,2)-1,4]$ \\
\hline
\end{tabular}

${ }^{*} \mathrm{p} \leq 0,10 ; * * \mathrm{p} \leq 0,05 ; * * * \mathrm{p} \leq 0,01$

WHOQOL-BREF : version abrégée en 26 items du questionnaire World Health Organisation - Quality of Life

a Modèle de régression univarié (régression logistique pour les indicateurs dichotomiques - les deux questions sur le bien-être général - et linéaire pour les indicateurs continus - les quatre scores de qualité de vie)

b Modèle de régression multivarié ajusté sur le sexe, la situation familiale, le fait d'avoir déjà exercé un autre métier, le niveau d'enseignement, le temps de trajet aller simple, le fait d'être à temps partiel,

d'enseigner dans $\geq 2$ établissements d'enseignement, la zone d'implantation et la taille de l'établissement, l'indicateur d'origine sociale des élèves, les trois scores du questionnaire de Karasek (latitude décisionnelle,

demande psychologique, soutien social) ainsi que le taux de satisfaction vis à vis des relations avec les élèves et avec les collègues.. 
Figure 1 : Distribution de l'âge des enseignants en fonction des catégories d'ancienneté ( $n=2320)$, enquête " Qualité de vie des enseignants ",

Fondation MGEN/Éducation nationale, 2013.

120

100

80

Ancienneté $\quad \square \leq 5$ ans $\quad \square-29$ ans $\quad \square \geq 30$ ans

20

0

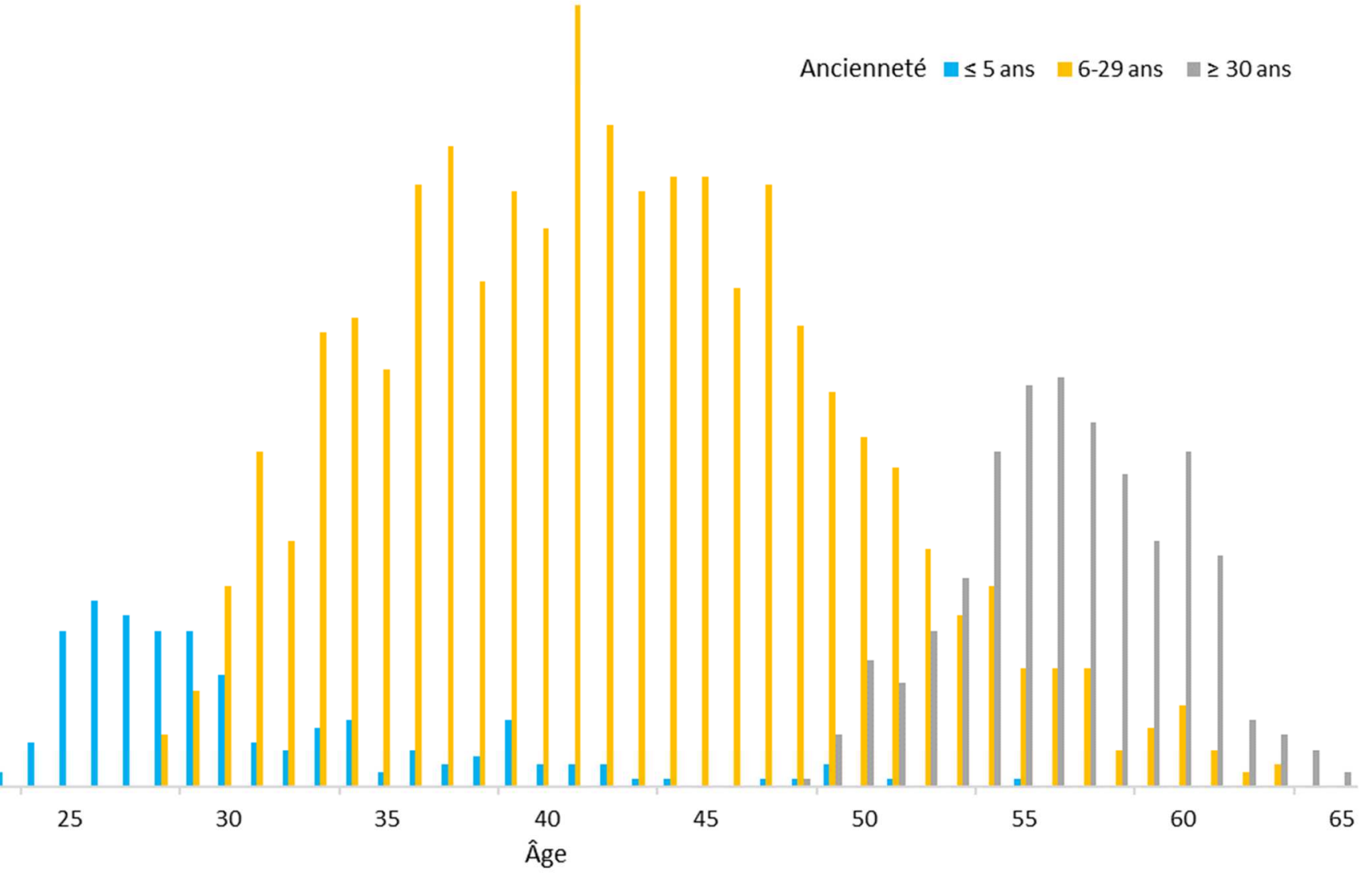


Figure 2 : Indicateurs de bien-être au travail en fonction de l'ancienneté, enquête " Qualité de vie des enseignants », Fondation MGEN/Éducation nationale, 2013.

\section{A. Bilan de l'expérience professionnelle}

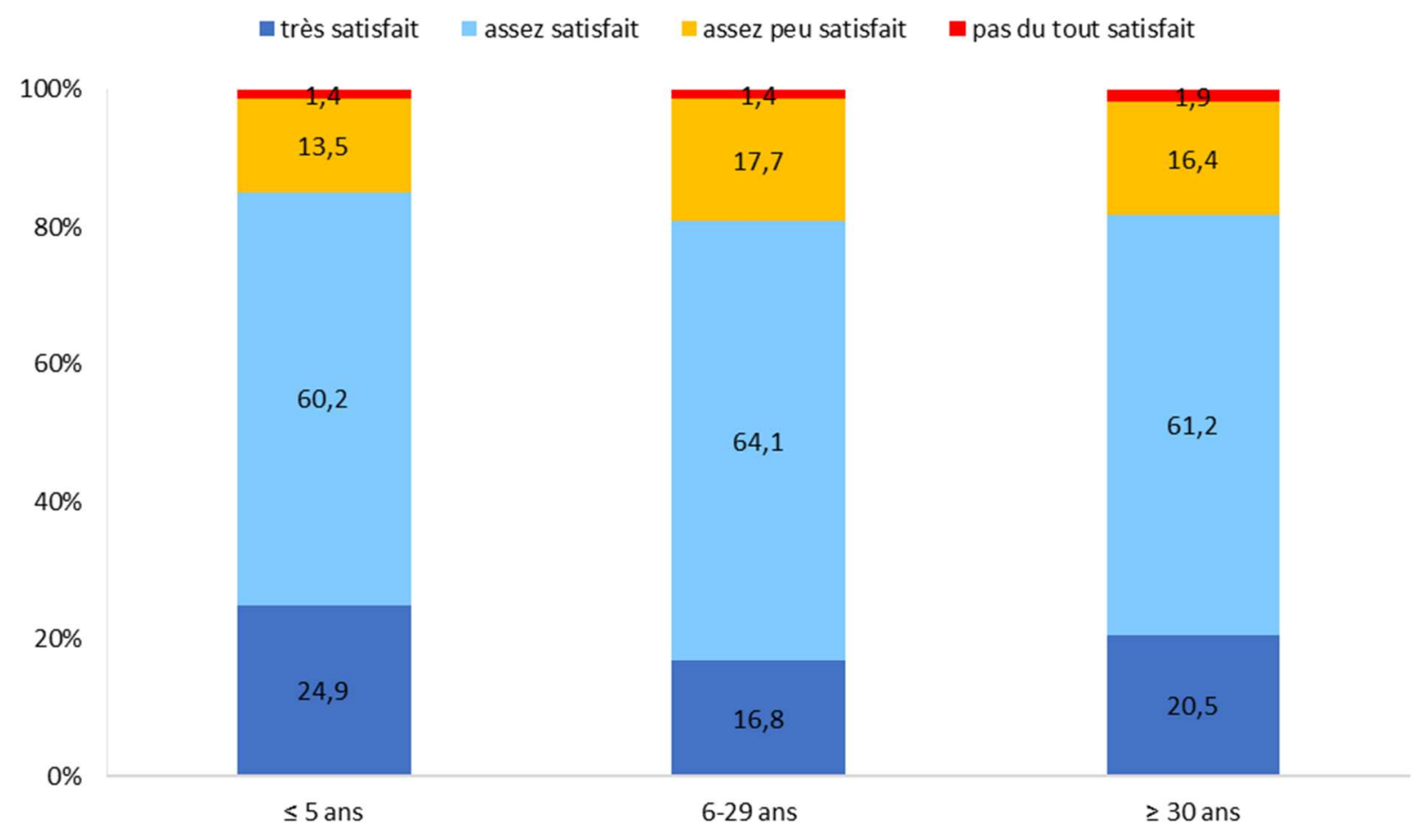

B. Evolution du métier depuis 5 ans

a de moins en moins difficile $\quad$ ni plus ni moins difficile $\quad$ de plus en plus difficile

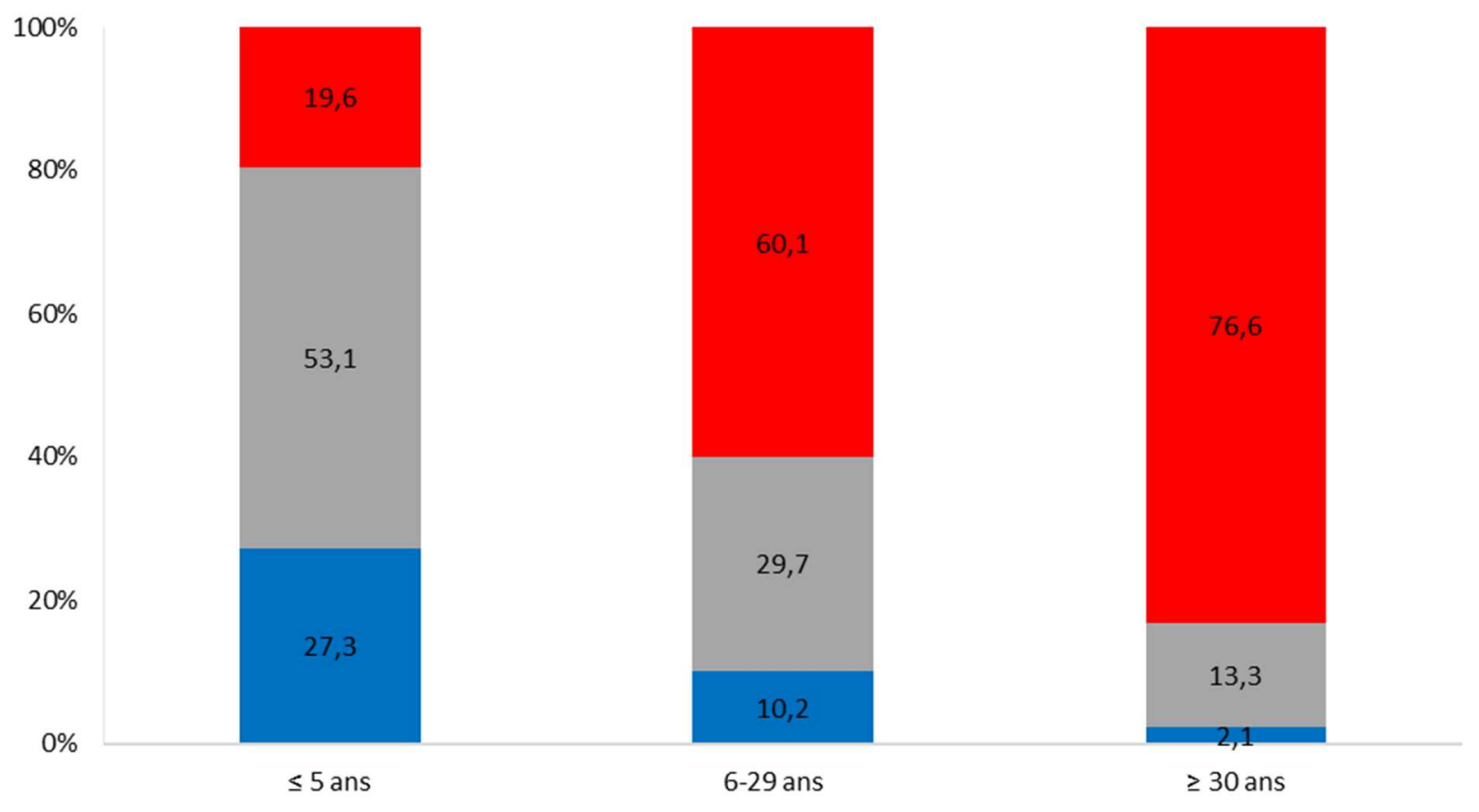


Figure 3 : Indicateurs de bien-être général (WHOQOL-BREF) en fonction de l'ancienneté, enquête "Qualité de vie des enseignants ", Fondation MGEN/Éducation nationale, 2013 WHOQOL-BREF : version abrégée en 26 items du questionnaire World Health Organisation - Quality of Life

A. Qualité de vie

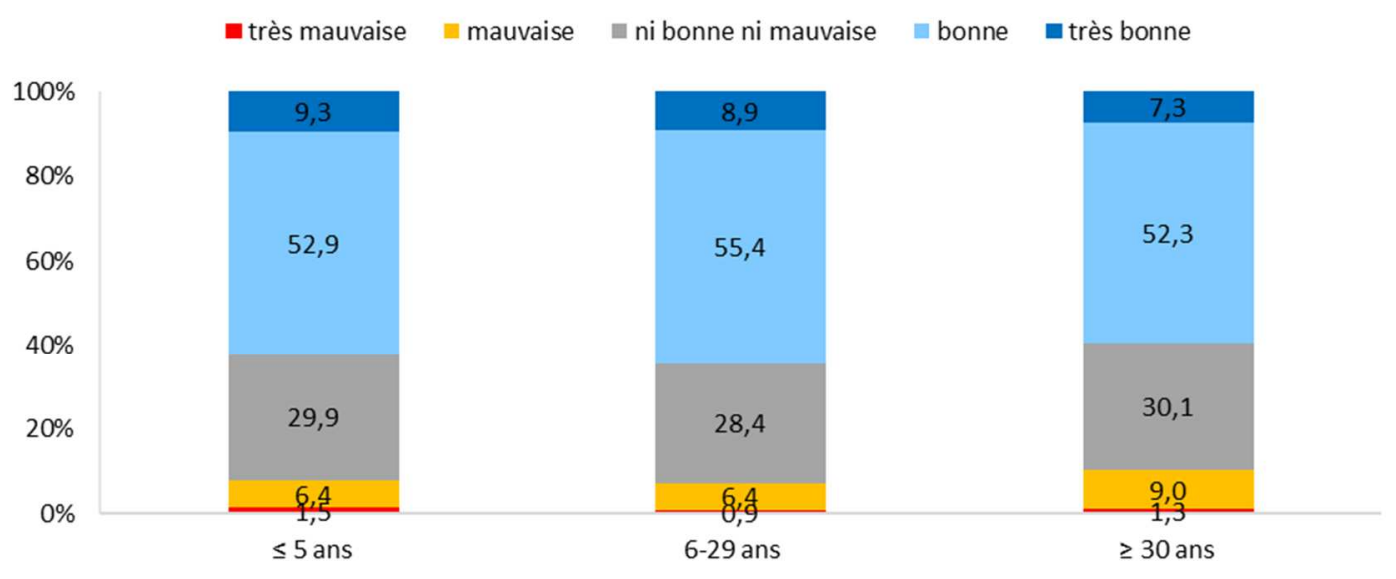

B. Santé perçue

m pas du tout satisfait $\quad$ pas satisfait $\quad$ ni satisfait ni insatisfait $\quad$ satisfait $\quad$ très satisfait

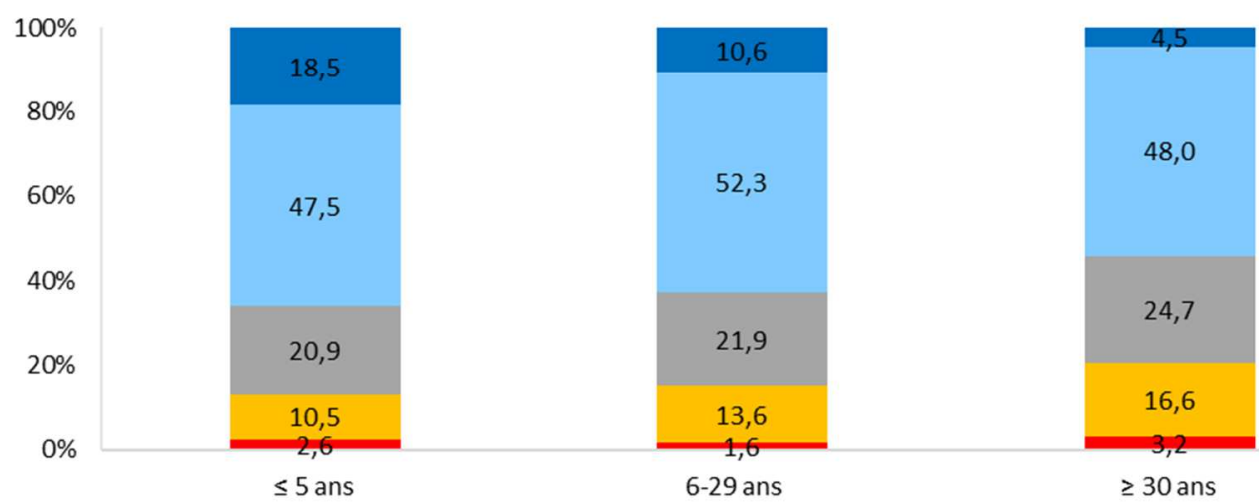

C. Dimensions de la qualité de vie

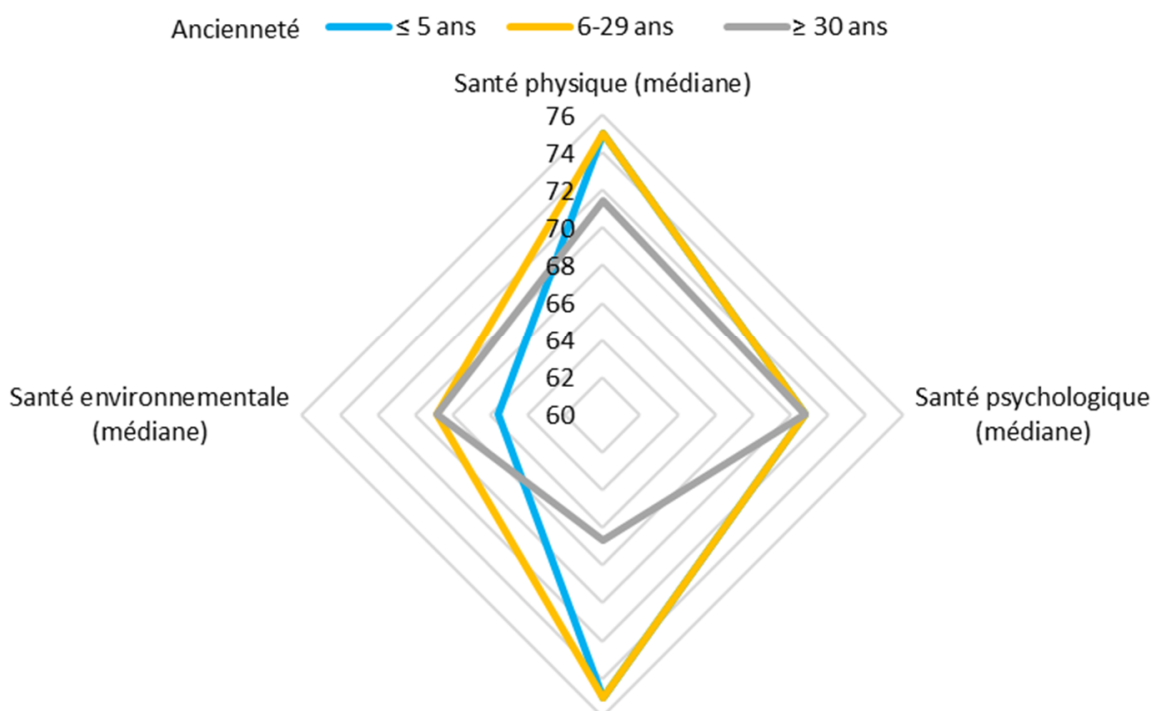

Relations sociales (médiane) 